\title{
A study on Prevalence of Potential Drug Drug Interactions in Community Pharmacies of Mysore city
}

\author{
Patel Jaskumar Nileshkumar ${ }^{1}$, Ansu Anie Sunny ${ }^{2}$, Jaidev Kumar ${ }^{3 *}$, \\ Mit Kaushikbhai Suthar ${ }^{4}$, Umesh ${ }^{5}$ \\ *Lecturer, JSS College of Pharmacy, SS.Nagar, JSS Deemed University, Mysore \\ jaidev.kumar9@gmail.com
}

\begin{abstract}
:
Introduction: A clinically significant interaction between two drugs is said to have occurred when the therapeutic or toxic effect of one drug is altered as a consequence of co-administration of another drug. The elderly populations are at increased risk because of decreased functioning of the physiological systems, presence of co-morbidities which require multiple medications for proper treatment. Medication safety is an important issue for the physician, pharmacist and other health care professionals.
\end{abstract}

Objectives: To determine the prevalence of prescriptions with drug-drug interactions among patients with chronic diseases.

Results and Discussion: Out of 800 prescriptions reviewed, we found 485(61\%) prescriptions without any potential drug drug Interactions and 315(39\%) prescriptions with at least one Potential drug-drug interaction. A total of 500 potential drug drug Interactions were identified during the study. The prevalence of prescriptions with potential drug drug Interactions among the study population was found to be $39.37 \%$.

Conclusion: The community pharmacists should be created more awareness about drug drug interactions by conducting educational workshop on drug drug interaction so that any type of drug drug interaction can be identified and appropriate management measures can be taken in according to the significance of drug drug interaction.

Keywords: Drug Drug Interactions, Prevalence.

\section{INTRODUCTION}

Drug-drug interactions (DDIs) have received a great deal of recent attention from the regulatory, scientific, and health care communities worldwide. A drug interaction is defined as the pharmacologic or clinical response to the administration of a drug combination differing from that anticipated from the known effect of the two agents. The interaction may result in a change in the nature or type of response to a drug (i.e., Pharmacodynamic interactions), or a change in the magnitude or a duration of response to a drug (i.e.,Pharmacokinetic interactions) ${ }^{1,2}$.A clinically significant interaction between two drugs is said to have occurred when the therapeutic or toxic effect of one drug is altered as a consequence of co-administration of another drug. ${ }^{3}$

Many studies have reflected polypharmacy as one of the major risk factor in occurence of PDDIs. Patient populations at high risk include the elderly, critical care patients and patients with co morbidities. The elderly populations are at increased risk because of decreased functioning of the physiological systems, presence of co-morbidities which require multiple medications for proper treatment. Medication safety is an important issue for the physician, pharmacist and other health care professionals. ${ }^{4,5,6}$

Many studies have reflected polypharmacy as one of the major risk factor in occurence of PDDIs. Patient populations at high risk include the elderly, critical care patients and patients with co morbidities. The elderly populations are at increased risk because of decreased functioning of the physiological systems, presence of co-morbidities which require multiple medications for proper treatment. Medication safety is an important issue for the physician, pharmacist and other health care professionals. ${ }^{4,5,6}$ 


\section{General objective}

- To assess the potential drug-drug interactions in prescriptions of patients with chronic diseases in community setting.

\section{Specific objectives}

- To determine the prevalence of prescriptions with drug-drug interactions among patients with chronic diseases.

\section{MeThodology}

This was cross sectional study carried out over a period of six months and it was approved by JSS Institutional of Human Ethical Committee to carry out this research work in different community pharmacies located in Mysore city.

\subsection{Data analysis}

\subsubsection{Patient Characteristics}

All the enrolled patients were grouped according to their age, gender, number of drugs prescribed and presence of comorbidities.

\subsubsection{Potential Drug-Drug Interactions}

Patients who experienced potential drug-drug interactions were categorized and analyzed separately.

Prevalence of PDDIs was calculated by using the following equation.

$$
\text { Prevalence of PDDIs }=\frac{\text { No.of Prescriptio ns with PDDIs }}{\text { Total number of prescription reviewed }}
$$

Severity of potential drug-drug interactions was assessed using Micromedex severity criteria and was categorized as minor, moderate and major interactions which were analyzed.The distribution of potential drug-drug interactions per patient was evaluated.

\subsubsection{Predictors of Potential Drug-Drug Interactions}

Patients with potential drug drug Interactions and patients without potential drug drug Interactions were grouped and compared according to their age, gender, number of drugs and presence of comorbidities.Continuous variables like age and number of drugs were presented as mean +/Standard Deviation. Categorical variables like gender and presence of chronic diseases with or without comorbidities were presented as number with percentage.

\subsection{Statistical Analysis Applied for Potential Drug Drug Interactions}

The predictors associated with the potential drug-drug interactions were identified at a $\mathrm{p}$ value of $<0.05$. Student t-test was performed to obtain $\mathrm{p}$ value for continuous variables. Chi-square test was performed to obtain $\mathrm{p}$ value for categorical variables.

\section{RESUlTS AND DisCUSSION}

\subsection{Prevalence of Potential Drug-Drug Interactions}

Out of 800 prescriptions reviewed, we found 485(61\%) prescriptions without any PDDIs and $315(39 \%)$ prescriptions with at least one Potential drug-drug interaction. A total of 500 PDDIs were identified during the study.

The prevalence of prescriptions with PDDIs was calculated by using the formula:

Prevalence $=$ No. of prescriptions with PDDIs $/$ Total No. of prescriptions reviewed

$$
=315 / 800=39.37 \%
$$

The prevalence of prescriptions with PDDIs among the study population was found to be $39.37 \%$. Similar result was observed in the study conducted by $\mathrm{H}$. Kafeel et al in Karachi, where the prevalence of PDDIs in prescriptions dispensed by the community pharmacies was $40 \%$. The prevalence of PDDIs is presented in figure no.1. 


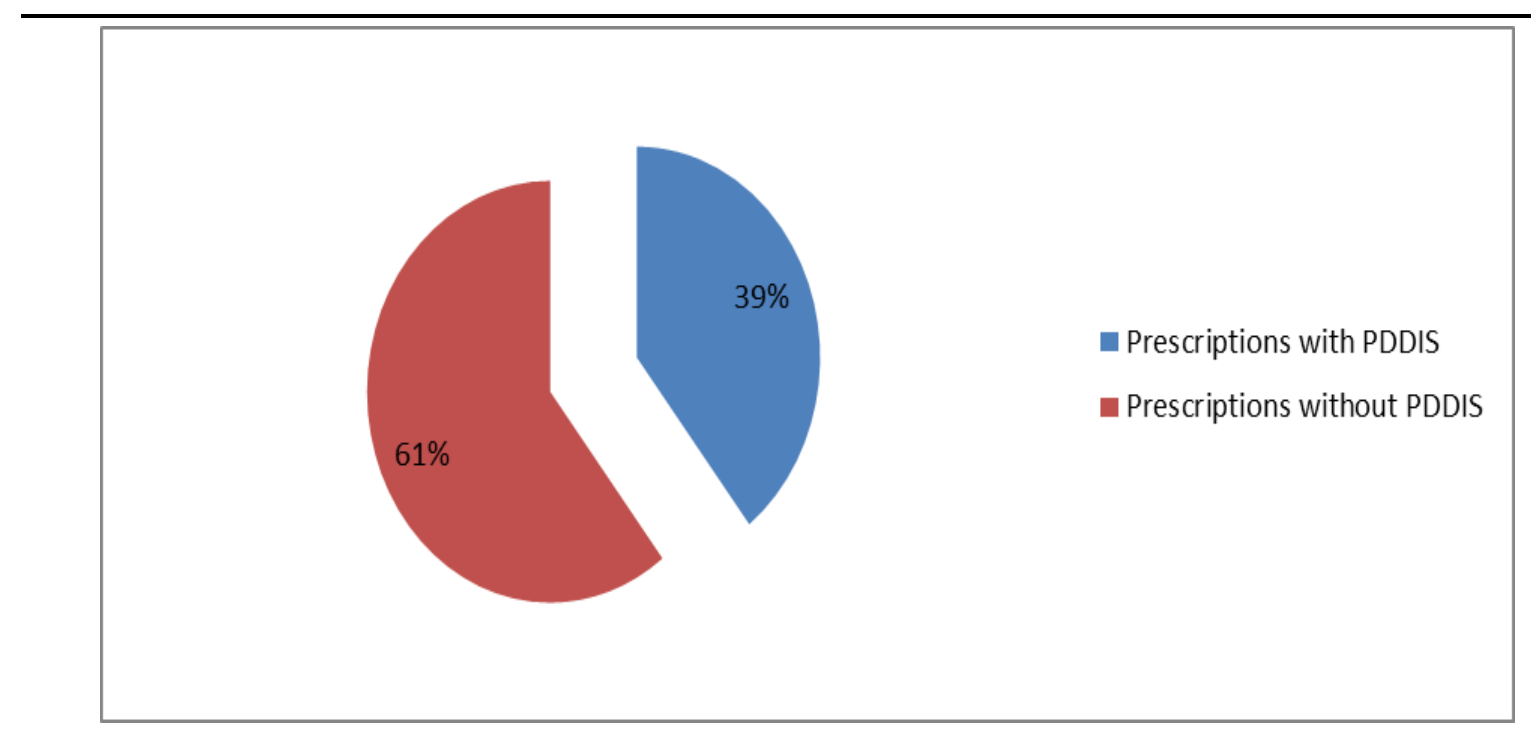

Figure1. Prevalence of PDDIs

\subsection{Distribution of Potential Drug-Drug Interactions/Patient}

Among 315 patients with PDDIs, 186(59\%) patients had only one PDDI in their prescriptions, 84(26.6\%) patients had two PDDIs, 31(9.8\%) patients had three PDDIs, $10(3.17 \%)$ patients had four PDDIS and 5(1.5\%) patients had five PDDIs in their prescriptions for chronic diseases. The distribution of PDDIs per patient is presented in figure no: 2

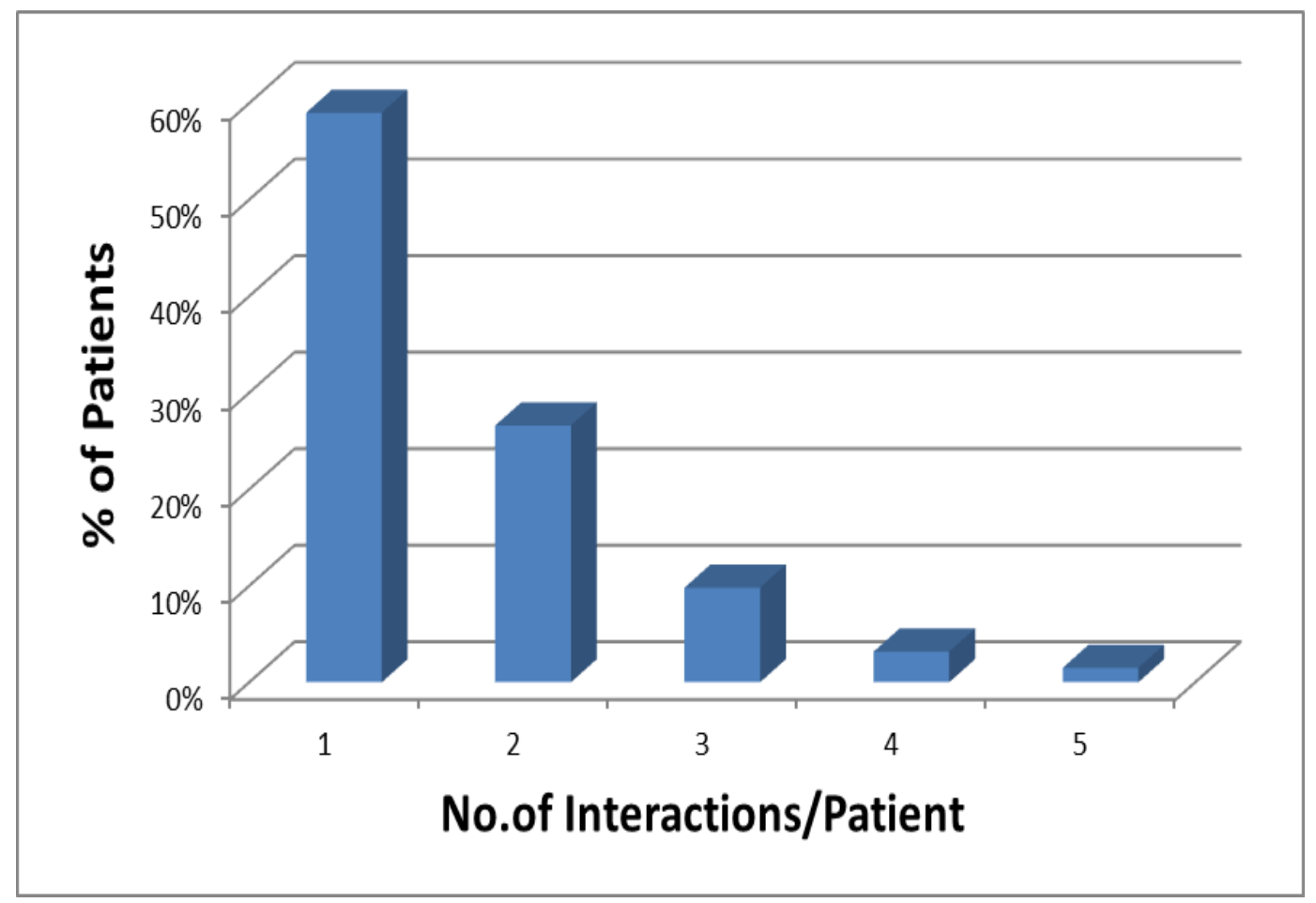

Figure2. Distribution of potential drug-drug interactions/Patient

\subsection{Chronic Diseases in Study Population}

Diabetes mellitus was the most commonly found chronic disease in our study population $(\mathrm{n}=507$, $63.3 \%)$, followed by hypertension $(\mathrm{n}=408,51 \%)$ and other cardiac disorders (ACS, CCF, arrhythmias; $\mathrm{n}=244,30.5 \%$ ). Less commonly found chronic diseases were neurologic disorders (Epilepsy, Alzheimer's Disease, Parkinson's Disease, neuropathy; $n=64,8 \%)$, asthma $(n=35,4.3 \%)$, psychiatric disorders (Schizophrenia, Depression, mania, BPAD; $n=32,4 \%)$, thyroid disorders $(n=22,2.7 \%)$, $\operatorname{COPD}(n=17,2.1)$, rheumatoid arthritis $(n=12,1.5 \%)$, tuberculosis $(n=7,0.8 \%)$ and CKD $(n=4$, $0.5 \%)$. The details of chronic disease in our study population are summarized in figure no. 3 


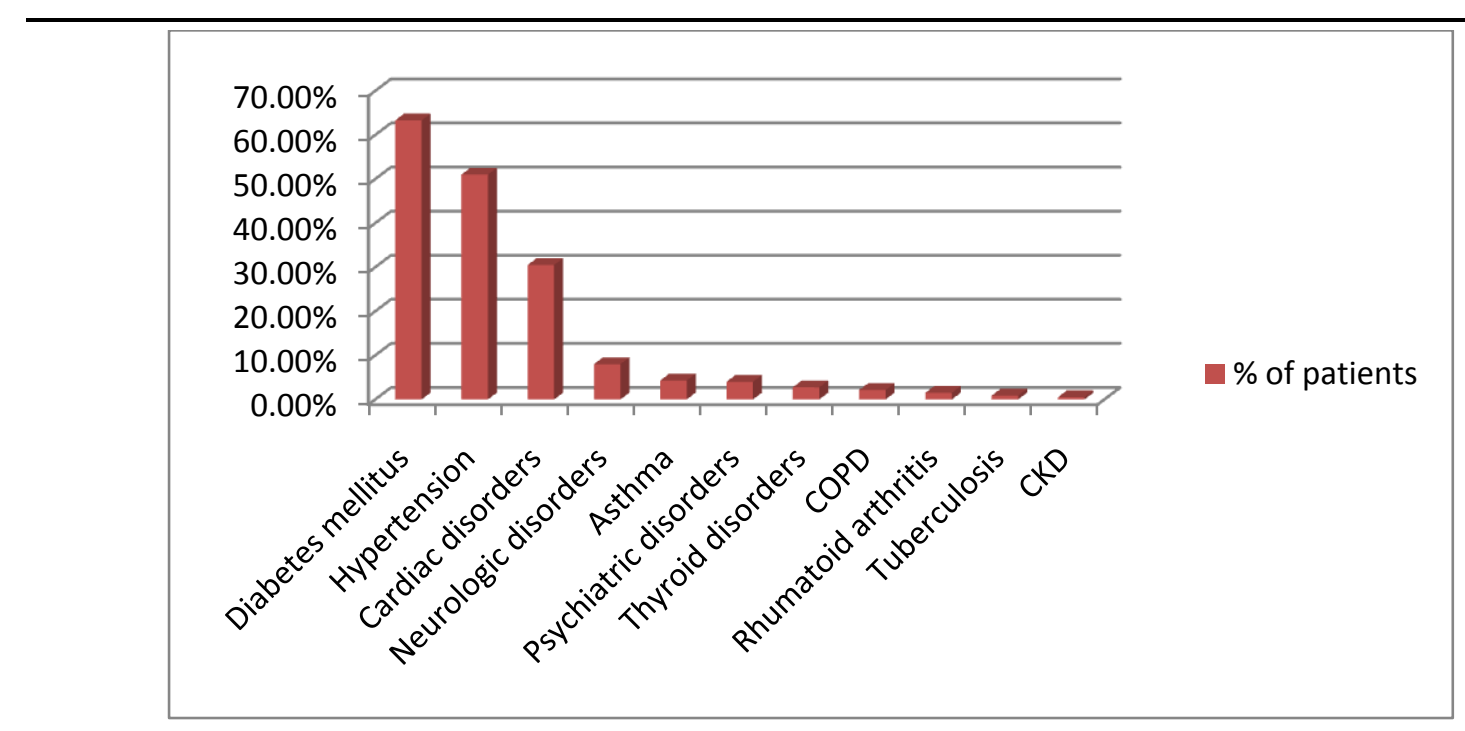

Figure1. Chronic diseases in study population

\section{Conclusion}

The study was conducted to assess the potential dug-drug interactions in prescriptions for chronic diseases dispensed in selected community pharmacies in Mysore city. The results of the study showed a 39\% prevalence of prescriptions with potential drug drug interactions among the 800 prescriptions reviewed. Anti-hypertensives and anti-diabetics were the commonly observed drug classes in potential drug drug interactions. Interaction between beta adrenergic blockers and glimepiride+metformin was the most commonly observed interaction in our study. Each and every prescription dispensed in community pharmacy should be screened for drug drug interaction.Failure to do regular screening of prescription in community pharmacy may end up with severe adverse drug reaction due to major drug drug interaction or it can end up with therapeutic failure either due to blockage of pharmacological effect and sometimes drugs acting as enzyme inducer will decrease therapeutic effectiveness of another drug by increasing metabolism and excretion .Therefore community pharmacists should be created more awareness about drug drug interactions by conducting educational workshop on drug drug interaction so that any type of drug drug interaction can be identified and appropriate management measures can be taken in according to the significance of drug drug interaction. The practicing community pharmacist should be motivated to have drug drug interaction resources such as text books, electronic software and important website references for tracing drug drug interactions. This type of research work done should reach message to all practicing community pharmacists in their respective places so that it can be be very helpful to minimise any major drug related problem associated with drug drug intateraction.

\section{FUTURE DiRECTIONS}

The research investigators have not created any awareness to practicing community pharmacist about drug drug interactions, therefore this research work undertaken in future should conduct educational workshop to practicing community pharmacist so that everyone can be more professional during their health care practice.

\section{REFERENCES}

[1] David S. Tatro, Drug interactions. In textbook of therapeutics. Edited by Eric T. Herfindal, Lippincott Williams and Wilkins. 8th ed., 2006; 47-72.

[2] Ben D Snyder,Thomas M Polasek ,Matthew P Doogue. Drug interactions: Principles and Practice. Aust Prescriber 2012;35:85-8

[3] Kafeel H, Rukh R, Qamar H, Bawany J, Jamshed M,Sheikh R, Hanif T, Bokhari U, Jawaid W, Javed Y,Saleem YM. Possibility of Drug-Drug Interaction in Prescription Dispensed by Community and Hospital Pharmacy. Pharmacology \& Pharmacy 2014 April 5: 401-7

[4] Bucşa C, Farcaş A, Cazacu I, Leucuta D, Achimas-Cadariu A, Mogosan C, Bojita M,How many potential drugdrug interactions cause adverse drug reactions in hospitalized patient s?. Eur J Intern Med. 2013 Jan;24(1):27-33. 
[5] Delafuente JC. Understanding and preventing drug interactions in elderly patients. Crit Rev Oncol Hematol. 2003 Nov;48(2):133-43

[6] Hajjar ER, Cafiero AC, Hanlon JT. Polypharmacy in elderly patients. Am J Geriatr Pharmacother. 2007 Dec;5(4):345-51.

[7] Stanton LA, Peterson GM, Rumble RH et al. Drug related admission to an Australian hospital. G.M. J Clin Pharm Ther 1994; 19:341-7.

[8] Daniel A. Hussar. Drug Interactions. In: Remington the science and practice of pharmacy. Edited by Alfonso R Geunaro. Lippincott Williams \& Wilkins. 21st ed., 2006;1:1889-1902.

[9] Mehuys E, Dupond L, Petrovic M, Christiaens T, Van Bortel L, Adriaens E, De BolleL, VanTongelenI, RemonJP, BousseryK.Medication management among homedwelling old er patients with chronic diseases: possible roles for community pharmacist. J Nutr Health Aging. 2012 Aug;16(8):721-6.

[10] Ansari J. Drug interaction and pharmacist. J Young Pharm. 2010 Jul;2(3):326-31.

[11] Luke Allen Miller, Karen Steinmetz Pater, Shelby Corman. The role of clinical decision support in pharmacist response to drug-interaction alerts. Res Social Adm Pharm. 2014 Oct 13.

[12] Chatsisvili A, Sapounidis I, Pavlidou G, Zoumpouridou E, Karakousis VA, Spanakis M, Teperikidis L, Niopas I. Potential drug-drug interactions in prescriptions dispensed in community pharmacies in Greece. Pharm World Sci.(2010) Apr;32(2):187-93. Epub 2010 Jan 14.

[13] Aparasu R, Baer R, Aparasu A.Clinically important potential drug drug interactions in community settings. Research in social and administrative pharmacy. Res Social Adm Pharm. 2007 Dec;3(4):426-37

[14] Ahmadizar F, Soleymani F, Abdollahi M. Study of drug-drug interactions in prescriptions of general practitioners and specialists in Iran 2007-2009. Iran J Pharm Res. 2011 Fall;10(4):921-31

[15] Bleich GW, Bleich A, Chiamulera P, Sanches AC, Schneider DS, Teixeira JJ. Frequency of potential interactions between drugs in medical prescriptions in a city in southern Brazil. Sao Paulo Med J. 2009 Jul; 127(4):206-10. 